\title{
PARTITIONS APPROXIMATED BY FINITE COSINE-SERIES
}

\author{
HARVEY DUBNER
}

\begin{abstract}
By using a specialized numerical Laplace transform inversion technique the number of partitions are expressed by a finite cosine-series. The accuracy of the results is only limited by the accuracy of computation and available computer time. The method is general and is applicable to all generating functions.
\end{abstract}

\section{INTRODUCTION}

In the late 1960s two papers appeared about numerical inversion of Laplace transforms $[3,1]$. Both papers were aimed at the engineering and probability world, where functions encountered were reasonably simple and 6-digit accuracy was more than adequate. Now, with the ready availability of powerful PC computers and excellent public-domain software, it was decided to reevaluate the utility of the power series expansion technique by applying it to the complicated generating functions found in partition theory.

The number of partitions can be expressed as a finite cosine-series with the accuracy of the results being limited only by the accuracy of computation. Because of this inherent accuracy it seems probable that there is some underlying theory yet to be discovered which would explain this behavior.

\section{OUTLINE OF THE METHOD}

Our objective is to find the coefficients of the power series represented by a given generating function. The outline of the theory of [1] which we will use is as follows:

1. From a given generating function $f(z)$ we define an auxiliary function $g(t)$ such that for integral values of $t, g(n)$ gives the coefficients of the power series of the generating function.

2. The Laplace transform, $G(s)$, of $g(t)$ is obtained trivially from $g(t)$.

3. The inverse Laplace transform, $g(t)$, of $G(s)$ is approximated by an infinite cosine-series.

4. Since only integral values of $t$ are needed, the infinite cosine-series can be converted to a finite cosine-series by using various symmetry and periodicity properties and introducing the generalized Riemann zeta function.

Received January 15, 1991; revised March 28, 1991.

1991 Mathematics Subject Classification. Primary 11P83; Secondary 41A58. 
5. Although the Riemann zeta function consists of an infinite number of terms, it is expandable asymptotically so that it can be calculated efficiently to a high degree of accuracy.

6. This finite cosine-series can be used to numerically approximate the coefficients in the power series of the generating function. The accuracy of the results is completely determined by the arithmetic accuracy of the computations and by the time available for the calculations.

\section{LAPLACE TRANSFORM OF A GENERATING FUNCTION}

We are given a generating function $f(z)$ which has a power series expansion,

$$
f(z)=\sum_{n=0}^{\infty} A(n) z^{n}
$$

Define a continuous function $g(t)$ such that $g(n)=A(n-1)$ with a straight line between $g(n)$ and $g(n+1)$, and $g(t)=0$ for $t<0$. The Laplace transform of $g(t)$ is

$$
G(s)=s^{-2}\left(1-e^{-s}\right)^{2} \sum_{n=0}^{\infty} A(n) e^{-n s} .
$$

Perhaps the easiest way to see that $G(s)$ is the Laplace transform of $g(t)$ is to consider $g(t)$ as the sum of a series of triangles with coordinates $(n, 0)$, $(n+1, A(n)),(n+2,0)$. The Laplace transform of each triangle is easy to derive, and (2) is the sum of these transforms. However, from (1),

$$
\sum_{n=0}^{\infty} A(n) e^{-n s}=f\left(e^{-s}\right) ;
$$

therefore,

$$
G(s)=s^{-2}\left(1-e^{-s}\right)^{2} f\left(e^{-s}\right) .
$$

This determines the Laplace transform of $g(t)$ trivially from the given function $f(z)$ by the transformation $z=\exp (-s)$. Then, having obtained $G(s)$, we invert it to find the function $g(t)$, which by virtue of its definition determines the coefficients

$$
A(n)=g(n+1) .
$$

\section{INVERTING THE LAPLACE TRANSFORM}

For generating functions encountered in combinatorial theory it is almost always impossible to find an exact inversion for (3). However, the methods of $[3,1]$ can be used to numerically invert these functions, but the question is whether the accuracy of the method is adequate when applied to complicated partition functions. What follows is a short summary of the inversion theory.

An approximation formula for the Laplace transform inversion integral is given by the following infinite series:

$$
g(t)=\frac{2 e^{a t}}{N}\left[\frac{1}{2} \operatorname{Re}\{G(a)\}+\sum_{n=0}^{\infty} \operatorname{Re}\left\{G\left(a+\frac{n \pi i}{N}\right)\right\} \cos \frac{n \pi}{N} t\right] .
$$


The error which results from replacing the integral by the infinite series is of the order $\exp (-a N)$, provided we restrict $t$ to the interval $0<t<N / 2$. In practice, selecting the largest value of $t$ determines $N$, which together with the desired error limit determines $a$.

There still is the difficulty that the series extends to infinity. Truncation error can be large and is usually beyond our control. However, by only requiring answers for integral values of $t$ and using various periodicity properties, the infinite series can actually be summed completely by making use of the generalized Riemann zeta function.

The relevant equations are

$$
\begin{gathered}
g(m)=\frac{2 \exp (a m)}{N} \sum_{n=0}^{2 N-1} C_{n} \cos \frac{n \pi m}{N}, \\
s=a+\frac{n \pi}{N} i, \\
C_{n}=\operatorname{Re}\left[S_{n}(a, N)\left(1-e^{-s}\right)^{2} f\left(e^{-s}\right)\right], \\
S_{n}(a, N)=\sum_{k=0}^{\infty} \frac{1}{(s+2 k \pi i)^{2}}-\frac{\delta_{0 n}}{2 a^{2}},
\end{gathered}
$$

where $\delta_{0 n}$ is the Kronecker delta function,

$$
N \geq 2 m
$$

and

$$
a=\frac{E}{N} .
$$

In [1] it is shown how (9) is related to the generalized Riemann zeta function, which in turn can be expanded in an asymptotic series involving the Bernoulli numbers,

$$
\begin{aligned}
z(2, w)= & \sum_{k=0}^{L-1} \frac{1}{(k+w)^{2}}+\frac{1}{(L+w)}+\frac{1}{2(L+w)^{2}}+\frac{1}{6(L+w)^{3}} \\
& -\frac{1}{30(L+w)^{5}}+\frac{1}{42(L+w)^{7}} \pm \cdots-\frac{3617}{510(L+w)^{17}}
\end{aligned}
$$

with an error in the order of $50 /(L+w)^{19}$. For $L=1000$, this gives the generalized zeta function to about 55 digits while only requiring the summation of about 1000 terms. Equation (9) can be rewritten as

$$
S_{n}(a, N)=-\frac{z(2, w)}{4 \pi^{2}}-\frac{\delta_{0 n}}{2 a^{2}},
$$

where

$$
w=\frac{n}{2 N}-\frac{a}{2 \pi} i .
$$

In practice, $N$ is selected based on (9); $E$ and $L$ are chosen based on error considerations (see below). Equation (8), with (11), (7), (12), (13), and (14), is used to calculate the coefficients $C_{n}$ of the cosine-series. Equations (6) and 
(4) are used to calculate the coefficients $A(n)$ of the power series of the given generating function.

\section{ACCURACY OF CALCULATION}

There are three sources of error in the above calculations:

1. The theoretical error inherent in the inversion method. One of the strong points of the method is its tight error control. This error is about $\exp (-E)$, and there is no theoretical limit for $E$. For $E=70$, the error is about $10^{-30}$.

2. The error in calculating the generalized zeta function. As shown above, this error can be kept quite small without using excessive computer time.

3. Error due to arithmetic precision limitations. This method requires highprecision calculations on complex numbers. Fortunately, there is a publicdomain program for PC's, called UBASIC, ideally suited to this problem. It can process up to 2600-digit numbers, and no special handling is necessary for the complex domain. It is also aimed at the number theory world with appropriate built-in functions. For a discussion of UBASIC see [2]. Since any desired precision is available, this error can be ignored.

In theory, $E$ can be made as large as one wants, making the error due to this source effectively zero. This would indeed be true except that there is an interaction between errors. In (6), the multiplying term $\exp (a m)=\exp (E m / N)$ grows exponentially with $E$. Since the answer must remain virtually the same as $E$ grows, the cosine-series sum must decrease exponentially with $E$. Thus, the errors introduced by the zeta function become proportionately larger.

Searching for maximums resulted in the following table based on the accuracy of the term corresponding to $m=N / 2$ in (6):

\begin{tabular}{rrr}
$E$ & $L$ & Accuracy \\
\hline 70 & 100 & 25 digits \\
90 & 500 & 34 digits \\
105 & 2000 & 39 digits \\
120 & 6000 & 45 digits
\end{tabular}

That is, if 25-digit accuracy were required then $L=100$, and $E=70$ would be an optimum choice. For $L=100$, increasing $E$ to 100 decreases the accuracy to 19 digits. Dropping $E$ to 50 decreases the accuracy to 18 digits.

\section{Attenuation}

When experimenting with this method, it soon becomes apparent that more accuracy is obtained when the target coefficients are more uniform. Often, a simple transformation can add several digits of accuracy with little computational penalty. In (1), replace $z$ by $t z$, so that

$$
f^{\prime}(z)=\sum_{n=0}^{\infty}\left[A(n) t^{n}\right] z^{n} .
$$

For $t<1$, the new coefficient in brackets has been attenuated by the factor $t^{n}$. To compensate, $g(m)$ in $(6)$ must be divided by $t^{n}$. One approach is to do a 
fast calculation at low accuracy, determine $t$ so that the answers will be more uniform, then do the final, more accurate calculation.

\section{UNRESTRICTED PARTITIONS}

The generating function for the number of unrestricted partitions of $n$ is [4, pp. 273-296],

$$
F(x)=\frac{1}{(1-x)\left(1-x^{2}\right)\left(1-x^{3}\right) \cdots}=1+\sum_{1}^{\infty} p(n) x^{n} .
$$

If the maximum $n$ of interest is $m$, then the infinite product in the denominator can be terminated after $m$ terms. The degree of this polynomial is $m(m-1) / 2$. For $m=500$, the degree is about 125,000 ! We applied our theory to compute $p(n)$ up to 500 .

From $m=500, N$ should be 1000 , and therefore 2000 terms in the cosineseries are required. Knowing that $p(500)$ has 22 digits, we set $E=70, L=$ 100 , and attenuation $=0.9$ (since $(0.9)^{500} \approx 10^{-23}$ ). The results are shown in Table 1. It took about one hour to do the calculations on a PC386, running at $20 \mathrm{MHz}$.

TABLE 1

Unrestricted partitions

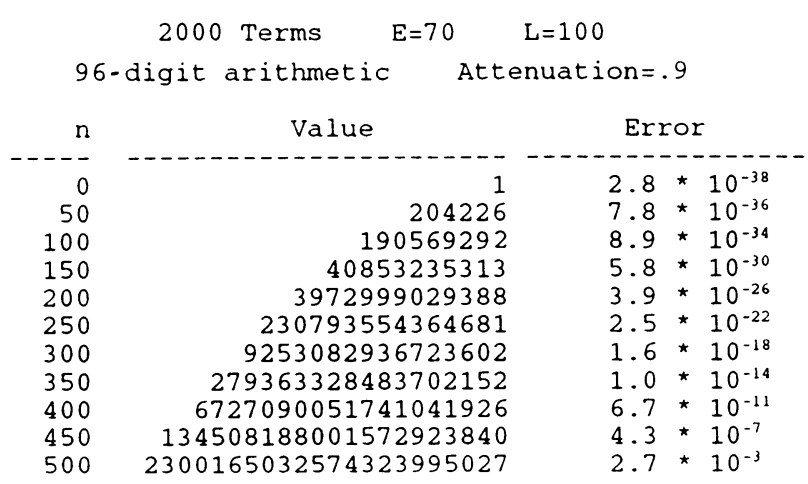

TABLE 2

Cosine-coefficients for unrestricted partitions

\begin{tabular}{cccc}
40 Terms & $E=70 \quad L=100$ & 96 -digit arithmetic & Attenuation $=1.0$ \\
$n$ & $C_{n}$ & $n$ & $C_{n}$ \\
\hline 0 & 0.0155834820 & --0 & -0.0146691001 \\
1 & 0.0550938561 & 20 & -0.0170261156 \\
2 & 0.0541519010 & 21 & -0.0190597232 \\
3 & 0.0524163367 & 22 & -0.0207842276 \\
4 & 0.0499495263 & 23 & -0.0222179896 \\
5 & 0.0468339096 & 24 & -0.0233824799 \\
6 & 0.0431664802 & 25 & -0.0243013537 \\
7 & 0.0390529749 & 26 & -0.0249995712 \\
8 & 0.0346023496 & 27 & -0.0255025890 \\
9 & 0.0299219682 & 28 & -0.0258356452 \\
10 & 0.0251137590 & 29 & -0.0260231517 \\
11 & 0.0202714114 & 30 & -0.0260882070 \\
12 & 0.0154785584 & 31 & -0.0260522300 \\
13 & 0.0108077954 & 32 & -0.0259347124 \\
14 & 0.0063203534 & 33 & -0.0257530816 \\
15 & 0.0020662418 & 34 & -0.0255226606 \\
16 & -0.0019152981 & 35 & -0.0252567095 \\
17 & -0.0055951495 & 36 & -0.0249665300 \\
18 & -0.0089535687 & 37 & -0.0246616146 \\
19 & -0.0119793746 & 38 & -0.0243498208
\end{tabular}


TABLE 3

Unrestricted partitions

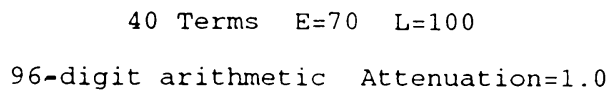

\begin{tabular}{rr}
$n$ & \multicolumn{1}{r}{ Value } \\
-0 & 1.00000 \\
1 & 1.00000 \\
2 & 2.00000 \\
3 & 3.00000 \\
4 & 5.00000 \\
5 & 7.00000 \\
6 & 11.00000 \\
7 & 15.00000 \\
8 & 22.00000 \\
9 & 30.00000 \\
10 & 42.00000 \\
11 & 56.00000 \\
12 & 77.00000 \\
13 & 101.00000 \\
14 & 135.00000 \\
15 & 176.00000 \\
16 & 231.00000 \\
17 & 297.00065 \\
18 & 385.57174 \\
19 & 980.00000 \\
20 & 422830.76599
\end{tabular}

Error
$--\cdot----$
$2.0 \star 10^{-38}$
$1.6 \star 10^{-37}$
$4.4 \star 10^{-36}$
$1.4 \star 10^{-34}$
$4.6 \star 10^{-33}$
$1.5 \star 10^{-31}$
$5.0 \star 10^{-30}$
$1.6 \star 10^{-28}$
$5.5 \star 10^{-27}$
$1.8 \star 10^{-25}$
$7.7 \star 10^{-24}$
$1.6 \star 10^{-21}$
$1.2 \star 10^{-18}$
$1.1 \star 10^{-15}$
$9.9 \star 10^{-13}$
$8.6 \star 10^{-10}$
$7.5 \star 10^{-7}$
correct answer $=385$
correct answer $=490$
correct answer $=627$

For small values of $n$, the calculations are accurate to about 40 significant digits. The accuracy systematically decreases to 24 digits as $n$ increases to 500 . Although the numbers change with the problem, the printout always looks like Table 1 when the answers are known to be integers. We repeated the same calculation with $E=105$ and $L=2000$. The accuracy at $n=500$ increased to 43 significant digits. However, the computer time increased to nine hours.

For illustrative purposes, the same calculation was made but with only 40 cosine terms. The cosine-coefficients (truncated) are listed in Table 2, and the partitions are shown in Table 3 . With 40 terms, high accuracy is assured only up to $n=10$, but we extended the calculations to 20 , with typical results. The cosine printout is also typical, but is not predictable (yet).

\section{MORE COMPLICATED FUNCTION}

There are more direct and faster methods for enumerating unrestricted partitions. However, this cosine-series method can be the most effective way to numerically evaluate other partition and combinatorial functions. For example, consider the number of ways $n$ similar objects can be distributed into $r$ different cells, including unrestricted partitions of the objects in each cell, a problem from graph theory. This is equivalent to the $r$-fold convolution of unrestricted partitions of $n$ objects in one cell.

The generating function for this is simply equation (16) raised to the $r$ th power,

$$
F(x)=\left(\frac{1}{(1-x)\left(1-x^{2}\right)\left(1-x^{3}\right) \cdots}\right)^{r} .
$$




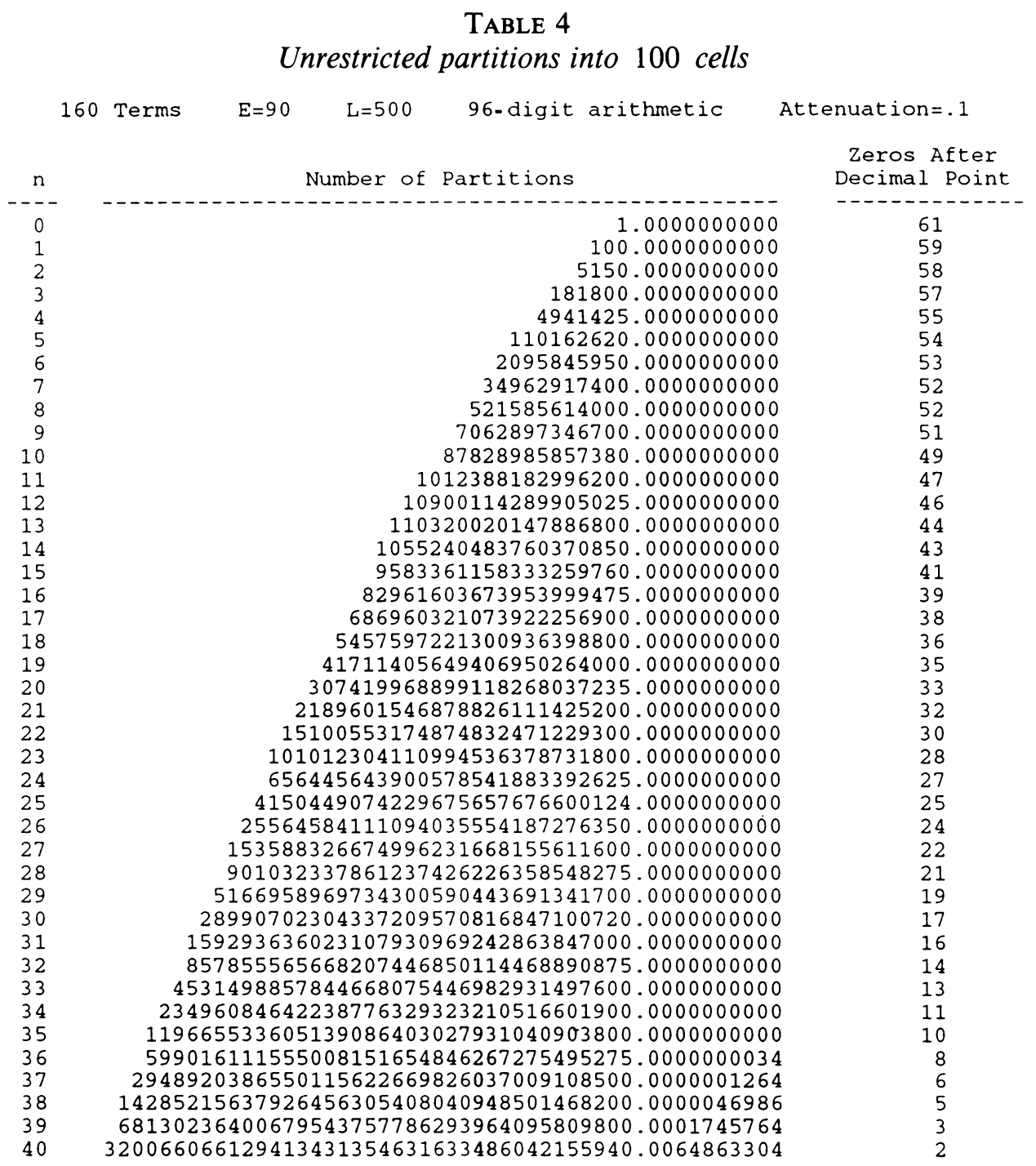

In the cosine method, this is accomplished by adding one instruction to the program, resulting in a negligible amount of additional computer time. The results are shown in Table 4 for $r=100$.

\section{Discussion}

This method for numerically evaluating generating functions by expansion into a finite cosine-series produces remarkably accurate results. It is even more impressive when one realizes that determining the number of unrestricted partitions of 500 is equivalent to calculating the five-hundredth derivative of a function, which includes a 125,000 th-degree polynomial, with 25 -digit accuracy.

As a special case, this method gives a closed-form expression for the number of partitions, restricted or unrestricted. Although this is an approximation, in 
the limit as one parameter $E$ becomes infinite, the expression becomes exact. This may be a fertile area for developing new reasonably simple expressions for partition functions.

\section{BIBLIOGRAPHY}

1. J. Abate and H. Dubner, A new method for generating power series expansions of functions, SIAM J. Numer. Anal. 5 (1968), 102-112.

2. C. Caldwell, The near repdigit primes, J. Recreational Math. 22 (1990), 101-109.

3. H. Dubner and J. Abate, Numerical inversion of Laplace transforms by relating them to the finite Fourier cosine transform, J. Assoc. Comput. Mach. 15 (1968), 115-123.

4. G. H. Hardy and E. M. Wright, An introduction to the theory of numbers, 5th ed., Oxford Univ. Press, Oxford, 1979.

449 BeVerly RoAd, Ridgewood, New Jersey 07450

E-mail address: 70372.1170@compuserve.com 